\title{
Application of the Base Transceiver Station with Smart Antennas in the Power Distribution Sector
}

\author{
B. A. Kleinau $\left(\mathbb{D},{ }^{1}\right.$ D. L. de Melo, ${ }^{2}$ M. T. de Melo $\left(\mathbb{D},{ }^{2}\right.$ D. C. P. Barbosa, ${ }^{3}$ A. J. B. Oliveira, ${ }^{2}$ \\ C. P. N. Silva, ${ }^{2}$ and J. M. A. M. de Oliveira ${ }^{4}$ \\ ${ }^{1}$ Companhia Energética de Pernambuco (CELPE)-Neoenergia, Recife, Brazil \\ ${ }^{2}$ Departamento de Eletrônica e Sistemas, Universidade Federal de Pernambuco (UFPE), Recife, Brazil \\ ${ }^{3}$ Departamento de Engenharia Elétrica, Universidade Federal de Pernambuco (UFPE), Recife, Brazil \\ ${ }^{4}$ Departamento Acadêmico de Controle de Sistemas Eletrônicos, Instituto Federal de Pernambuco (IFPE), Recife, Brazil
}

Correspondence should be addressed to B. A. Kleinau; brunoagra@gmail.com

Received 14 October 2020; Revised 19 May 2021; Accepted 12 June 2021; Published 18 June 2021

Academic Editor: Ahmed Toaha Mobashsher

Copyright ( $\odot 2021$ B. A. Kleinau et al. This is an open access article distributed under the Creative Commons Attribution License, which permits unrestricted use, distribution, and reproduction in any medium, provided the original work is properly cited.

\begin{abstract}
The use of distribution automation systems has grown significantly in electricity companies in recent years. The main reason for this is the need to have a smarter network, in order to reduce the time of power interruption. For such applications, communication using radio frequency is preferred because this solution is more reliable than $3 \mathrm{G} / 4 \mathrm{G}$ and cheaper than fiber optics. In this context, it is of utmost importance to have a more efficient base transceiver station (BTS) that can cover the communication of a larger area. In this paper, the mathematical concepts of an intelligent antenna array, as well as the logical operation of an intelligent radiation system controller, are presented. Such system receives as inputs the geographic coordinates of network elements and automatically feeds an intelligent Yagi-Uda antenna array with the appropriate parameters, in order to optimize the radiation pattern into the desired directions. The presented model uses a stochastic optimization method to automatically achieve a set of optimal electrical parameters to excite the array and efficiently direct its beams in a fully controlled way. Thus, the results obtained indicate that the proposed intelligent scheme allows the energy optimization of the antenna system, reducing in $61 \%$ the number of BTS needed to cover the same area, when compared to traditional collinear antenna systems.
\end{abstract}

\section{Introduction}

Automation systems have been widely used by the electrical industry in recent years [1]. Currently, there is a considerable growth in the number of automated equipment, where radio frequency $(\mathrm{RF})$ communication is one of the main means of remote control used by companies to drive remote devices $[2,3]$. For this application, radio frequency communication is more reliable than $3 \mathrm{G} / 4 \mathrm{G}$, due to its independence of third-party operators, and cheaper than fiber optics, being, therefore, the most viable alternative $[4,5]$. In this context, it is extremely important to have more efficient BTS capable of operating devices distributed over a larger area.

Smart antennas use an array whose elements are antennas of low gain that are connected by a combination device [6]. Smart antennas can help in improving the communication system performance by increasing channel capacity and spectrum efficiency, extending range coverage, steering multiple beams to track many mobiles, and compensating electronically for aperture distortion [7]. For the most part of BTS solutions used in the electrical sector, the $\mathrm{RF}$ signal is propagated by a collinear array with an omnidirectional propagation [8]. In the location where the researches generated this article (Pernambuco, Brazil), an antenna array of collinear dipoles is used by the electricity distribution company (Neoenergia) in each of the Base Transceiver Stations. These arrays generally generate an omnidirectional radiation pattern with gain of about $6 \mathrm{dBi}$. By law, the Brazilian National Telecommunications Regulatory Agency (ANATEL-Agência Nacional de Telecomunicações) narrows the use of radio frequency communication for remote sensing directed at public 
interest service provider companies to the frequency ranges of 458 to $460 \mathrm{MHz}$ and 468 to $470 \mathrm{MHz}$. The first waveband is allocated to outstation transmission, whereas the second one is allocated to BTS transmission. Using these two bands, the communication protocol between the BTS and its outstations works in a half-duplex master-slave manner. Therefore, the BTS communicates with only one device at time in a pooling architecture, and the device only report to its manager BTS when it is questioned.

It is well known that several factors, such as the distance, vegetation, civil construction, and the terrain topology between the outstations and its manager BTS, can affect the signal link quality $[9,10]$. It can moreover generate disruption in the radio communication, leaving some of these outstations in shadow regions [11]. In order to overcome the path obstacles and then restore the communication link, it is necessary to increase the signal intensity, within the allowed power emission limits. Hence, for an optimized use of the power of the RF signal generator, it is convenient that the maximum power of the radio signal can be directed intelligently to certain interesting areas in a controlled way.

An alternative to achieve an adjustable directivity of the beams without the need of mechanical schemes is using smart antenna arrays. Using multiple antenna elements enables such arrays to produce the desired radiation characteristics [12], allowing to point the beams in the desired direction $[13,14]$. Antenna arrays have been widely adopted in several solutions, such as estimation of the direction of arrival (DOA) for the signal received by the antenna $[15,16]$ and concentration of the radiation on a predefined spot, reducing the energy wasted in directions where it is not required [17].

To increase the efficiency and reach of Radio Base Stations, it is presented in this paper the theoretical and constructive principles of a RF system that uses a smart Yagi-Uda antenna array with 360-degree coverage electronically controlled. The proposed system receives as input the geographical coordinates regarding the locations of the transmitter and the equipment to be driven, and through an embedded mathematical optimization model, it is able to automatically control the optimal radiation direction of the $\mathrm{RF}$ generator power, increasing the range and efficiency of a common BTS. The great advantage of this process is that it provides a high gain concentrated radiation beam in any angular direction of a remote device the BTS needs to communicate with.

\section{Antenna Array Theoretical Background}

An antenna array is the name given to the controlled grouping of antennas built in order to achieve gain and radiation diagram specifications that are unreachable if it is employed by a single antenna. It can be understood, therefore, as a new and complex antenna generated by the combination of a set of simpler antennas.

There are 5 possible mechanisms for the control of the antenna array radiation pattern [18]:

(1) Geometric configuration of the array (linear, circular, and planar)
(2) Relative separation between elements

(3) Relative diagram of individual elements

(4) Amplitude of excitation of each element

(5) Electrical excitation phase of each element

The first 3 mechanisms are related to geometric factors, while the latter are related to electrical factors. The main objective is to initially define the used geometric factors and then perform intelligent control by changing only the electrical factors.

For the application adopted for energy utilities, the best type of array to be applied is the linear, since the existing structures are already adapted for this type of antenna array. Other geometries would need physical and operational adaptations from the Operation and Maintenance teams.

It is also important to define the radiation pattern in terms of the antenna element type and the geometric configuration of the array. In punctiform antennas (with isotropic source) systems, for example, the total diagram of the array is equal to the diagram of a single antenna element multiplied by the array factor diagram (AF) [18]. Considering a collinear array with $\mathrm{N}$ isotropic elements with the same excitation amplitude and a gradual increase in the electric phase $B$ of excitation, as shown in Figure 1, the expression for the array factor is given by [19]

$$
\begin{aligned}
\mathrm{AF}= & 1+e^{+j(k d \cos \theta+\beta)}+e^{+j 2(k d \cos \theta+\beta)}+\cdots \\
& +e^{+j(N-1)(k d \cos \theta+\beta)}
\end{aligned}
$$

where $d$ is the spacing between antennas. It also may be written as

$$
\mathrm{AF}=\sum_{n=1}^{N} e^{j(n-1) \psi},
$$

with

$$
\psi=\mathrm{kd} \cos \theta+\beta .
$$

Considering that the maximum array factor occurs when $\psi=0$, regardless of the applied electrical amplitude being uniform or not, the maximum of the function will occur at an angle $\theta_{o}$, according to equation (3), when

$$
\beta=-\mathrm{kd} \cos \theta_{0}
$$

where $\beta$ is the electrical excitation phase that is gradually applied to the elements [17]. This mathematical method, known as phase shift model in [19], allows a control of the desired angle to direct the maximum power for arrangements that use omnidirectional elements.

However, when the array elements are directive, the phase shift model application proves inefficient, as shown in Figure 2. These patterns were obtained by the attempt of using the aforementioned method to direct the radiation pattern of the Yagi-Uda array in Figure 3 to $\theta_{0}=60^{\circ}$ and $\theta_{0}=30^{\circ}$. The positive direction of the $y$-axis (in green color) in Figure 2 points to $90^{\circ}$ of these generated azimuthal diagrams.

The Ansys HFSS [20] was the electromagnetic simulation software used for designing the antenna array presented in 


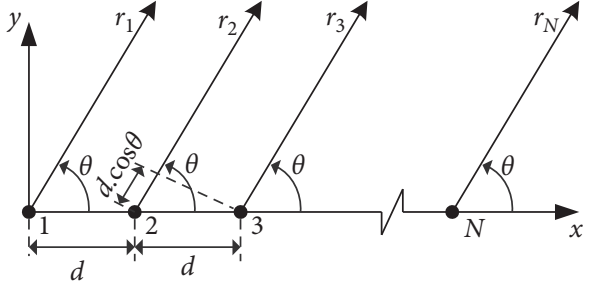

Figure 1: Antenna array for $N$ isotropic elements.

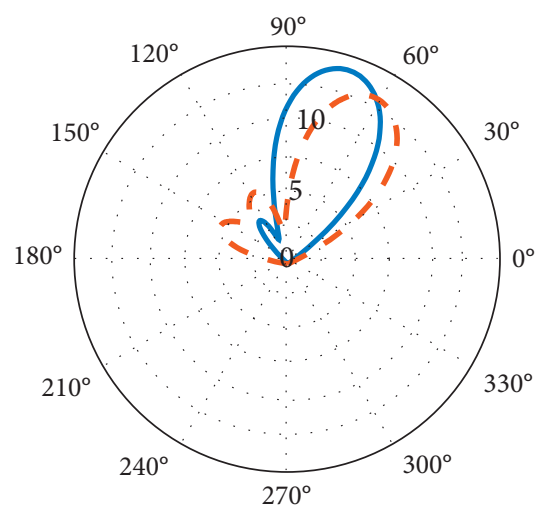

FIGURE 2: Resulting azimuthal diagrams of applying the phase shift mode to the Yagi-Uda array of Figure 3, with the purpose to point the beam to $\theta_{o}=60^{\circ}$ (continuous line) and $\theta_{o}=30^{\circ}$ (dashed line).

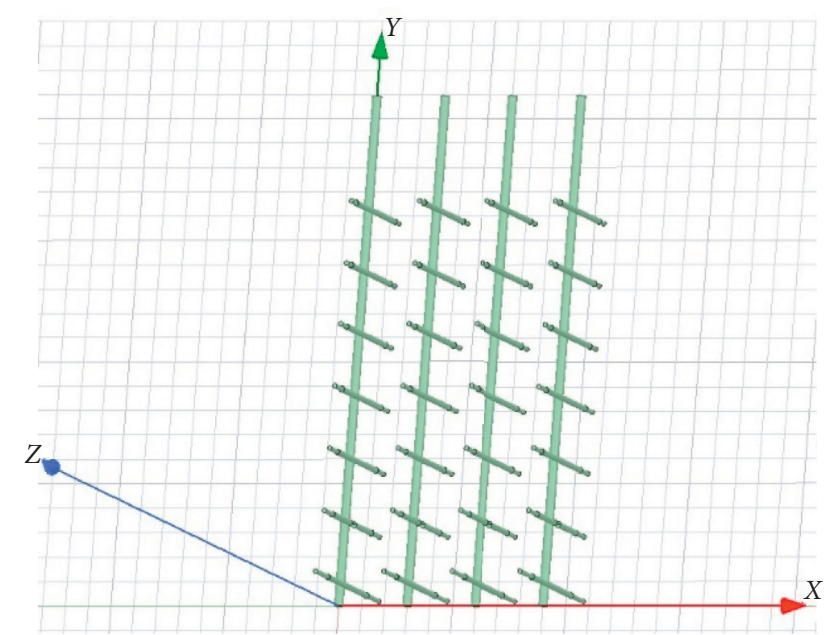

FIgURE 3: The 4 Yagi-Uda antenna array model. Each antenna has 7 elements.

Figure 2, as well as simulating it to get its radiation patterns. The HFSS is able to use both Finite Element Method (FEM) [21] and Method of Moments (MOM) [22] to solve electromagnetic problems. In all the simulations reported here, a boundary condition that uses the FEM as the numerical technique to perform its infinite element analysis is selected.

It is known that when the array element is directive instead of omnidirectional, it provides a greater gain in its physical oriented direction. In this case, to direct the beam to different directions, it is necessary to adopt some optimization method in order to find the proper sets of complex antenna excitations to the array, since the phase shift model shall not apply.

Elements of antenna arrays are spatially sampled and reradiate the incident fields that interact with the other elements causing the antennas to be mutually coupled [23]. Such mutual coupling can seriously degrade the performance of array signal processing methods, including beamforming techniques [24]. In order to minimize the interference among arrays and allow them to operate independently, the relationship $r \geq 2 D^{2} / \lambda$ must be met, where $D$ is the largest dimension of the array, $\lambda$ is the wavelength, and $r$ is the distance between two arrays [19]. Figure 4 shows the real example and the best result for $D$ for the experimental setup used to validate the model in the field measurements.

\section{Method Using the PSO Optimization}

Due to the need for more accuracy, Yagi-Uda antenna array models tend to set aside the use of array factor concepts (equations (1) and (2)). Being mathematically expressed through the induced mutual impedances [25], the electromagnetic interferences between their elements make almost mandatory the use of more robust numerical approaches in its modeling. Examples of such numerical costs are the application of Finite Element Method (FEM) [26], Green Discrete Functions (FDG-FTDT) [27], and The Method of Moments (MOM) [28].

In order to calibrate isotropic or even Yagi-Uda antenna array parameters, the literature currently has been using stochastic optimization techniques [29-33]. These stochastic computing methods (e.g., Particle Swarm Optimization) help to overcome these complexities in the equation of the model concerned [30]. This section addresses the use of the Particle Swarm Optimization Method (PSO) with the aim to direct the beam of maximum power to $\theta_{o}$ in the radiation diagram of the Yagi-Uda antenna array, as well as minimize its secondary lobes. The array electrical factors are handled in the method for these purposes.

Having its initial version developed by Kennedy and Eberhart [34], the PSO method seeks a parameter adjustment that better accommodates the mathematical system modeling to the restrictions imposed by the chosen objective function. Its concept was initially developed by applying a population-based artificial life (A-life) approach to evolutionary computation with the aim of optimizing continuous nonlinear functions [34]. As an evolutionary self-adaptative algorithm, the advances of its independent agents through the iterations emulate the synchronism of birds in a flock or bees in a swarm.

Each method possible solution, referred commonly to as a particle, has its trajectory continuously adjusted towards its own best location achieved so far and towards the position of the best particle of the entire swarm at each time step (generation). The individual influence component of a particle is called its Pbest (personal best) component, while the social influence component of the particle set is commonly referred to as its Gbest (global best) [34-36]. Depending on the weight values appended to these 


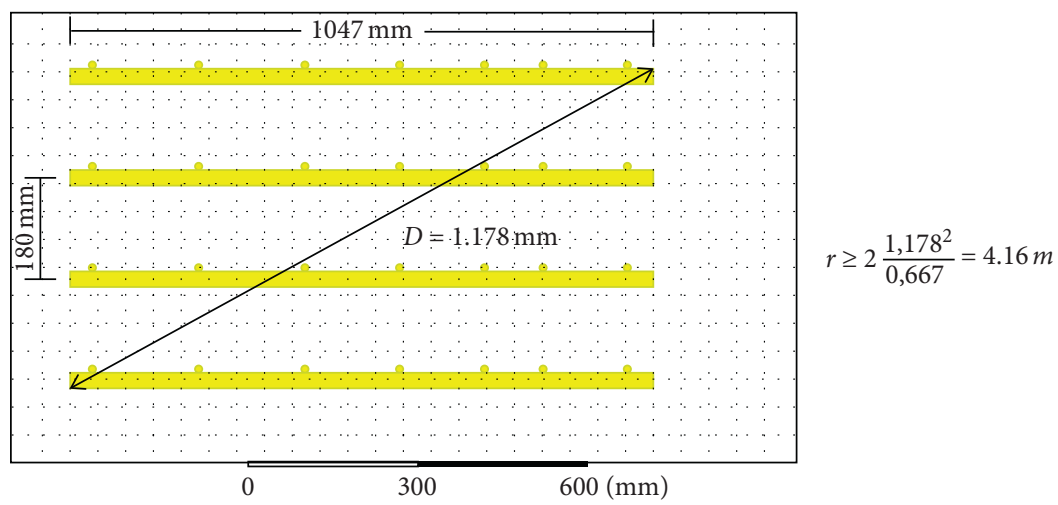

FIGURE 4: Calculation to define the distance required to avoid mutual coupling.

components, the velocity of each particle can be dominated by its own flying experience or by the flying experience of the other particles [35]. The third and last component used to the particle position update is called the momentum component and it uses the previous computed particle velocity to provide the necessary inertial factor that the particle needs to mimic a natural fluid motion to roam across the search space [37].

Figure 5 shows an example of the PSO particle's arrangement in four different progressive stages during the iteration algorithm process in a $R^{2}$ search space. The dashed arrows indicate the particles current movement direction, whereas the global optimum position, which the method is looking for by using some objective function, is indicated in red color.

In this work, the variables, which the method seeks to find an optimal configuration, are the electrical excitations in the antennas of the array. By this configuration, it is hoped to obtain an improved pattern diagram. Thus, each PSO particle is a point contained in a subspace $\Omega \subset R^{8}$, where four of its components are the power amplitudes $(\epsilon[0 ; 1]$, in Watts) and the other four are the signal phase shifts with which the antennas are fed, $\in[0 ; 2 \pi]$.

The position update of each particle is built based on the traditional algorithm, found in the literature ([34, 38]), namely,

$$
\begin{aligned}
v_{i}(k+1)= & c_{1} \cdot \operatorname{rand}_{1} \cdot\left(p_{\text {ibest }}(k)-x_{i}(k)\right) \\
& +c_{2} \cdot \operatorname{rand}_{2} \cdot\left(g_{\text {best }}(k)-x_{i}(k)\right)+w \cdot v_{i}(k), \\
x_{i}(k+1)= & x_{i}(k)+v_{i}(k+1),
\end{aligned}
$$

with $w$ being the moment of inertia and $c_{1}$ and $c_{2}$ being the cognitive and social dynamic accelerations, respectively. These PSO parameters are updated through iteration by

$$
\begin{aligned}
& w=\left(w_{\min }-w_{\max }\right)\left(\frac{k}{K_{\max }}\right)^{2}-w_{\max }, \\
& c_{1}=c_{1 \max }-\left(c_{1 \max }-c_{1 \min }\right) \cdot \frac{k}{K_{\max }}, \\
& c_{2}=c_{2 \max }-\left(c_{2 \max }-c_{2 \min }\right) \cdot \frac{k}{K_{\max }},
\end{aligned}
$$

while the maximum computed speed is maintained, by means of saturation, in the interval $\left(-v_{\max }, v_{\max }\right)$, with

$$
v_{\max }=\left(\left(K_{v}-\left(\frac{k}{K_{\max }}\right)^{p}\right) V_{\max }\right),
$$

where $k$ is the numerical value of the current iteration and $K_{\max }$ is the proposed total number of iterations. $w_{\min }, w_{\max }$, $c_{1 \text { max }}, c_{1 \text { min }}, c_{2 \max }, c_{2 \text { min }}, K_{v}, p$, and $v_{\text {max }}$ are positive constants adjusted by trial and error, following the initial selection criteria pointed out by $[35,39,40]$. The reflecting condition is used as the boundary handling condition to the proposed optimization. The reflecting, invisible, and damping boundary techniques are three promising methods when used in the PSO for handling antenna calibration problems [36, 41, 42].

The objective function used in the PSO contains two components. The first one maximizes the value of the diagram in the angular position $\theta_{o}$, where it is intended to maximize the power. The second one, with lower weight, minimizes the secondary lobes $[37,43]$ in positions over to 0 $\mathrm{dBi}$, what rectifies some adverse events arisen by the first one. Each particle has its components injected into the mathematical model of the antenna array, and the errors resulting from these two objectives are calculated and added. The resulting value then is used as a performance meter for the current particle's position.

In order to find the secondary lobes in the pattern diagram generated from the position of each particle, a lobes detector code is written. It is capable of returning the angular positions in which the lobes of interest are, namely, those ones bigger than $0 \mathrm{dBi}$. By these positions, the respective values in $\mathrm{dBi}$ are taken in the diagram. Then, these error values are added together resulting in the weightless second objective function's component. Lobes that have a position near the desired lobe are not computed in this sum, that is, those ones angularly closer than $4^{\circ}$ from $\theta_{0}$. This approach allows the pattern to accommodate itself in a configuration, within a confidence interval that minimizes the error.

The applied PSO algorithm was written in Python [44] and used by access remote to the HFSS [20]. For each algorithm iteration, the current position of each particle is inserted in the HFSS design as its electrical excitations, using the proper Python packages and modules. Soon after it, the 

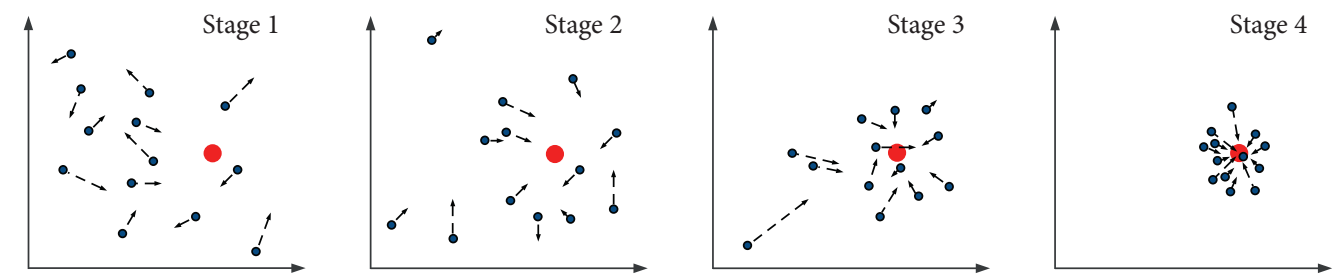

Figure 5: Particle's arrangement in the PSO in four different progressive iteration instants. The red circle indicates the global optimum location.

resultant radiation patterns are extracted from the HFSS by the algorithm, exported as a table containing its set of points. Then, the objective function uses these tables to score each particle position as mentioned above. Figure 6 presents a flowchart that shows the algorithm generic process of remotely accessing the HFSS in order to score a particle position.

\section{Computational System Description}

The complete system consists of an antenna array formed by a symmetrical circular set of 3 antenna arrays shown in Figure 7 , with an individual control of the excitation amplitude and the electrical excitation phase for each individual element.

An antenna array controller (AAC), based on [45], is responsible for all the processing intelligence to define the parameters which will excite the array so that the maximum gain is pointed towards the desired direction. In its internal memory, there is an inserted table containing all the optimal electrical excitations sets for each azimuthal direction, obtained by the application of the PSO to the simulated antenna array model.

The geographical coordinates of the BTS, where the smart antenna array is located, as well as the geographical coordinates of the remote devices to be controlled are the input parameters of the AAC. With the information of the location of the BTS and the devices, the controller converts the geographic coordinates to polar coordinates identifying the angle $\theta_{0}$. Such angle corresponds to the relative direction of the device intended to receive the maximum power of the BTS.

With $\theta_{0}$ obtained, the AAC catches its corresponding set of electrical feeding at the PSO optimal excitation table. Then, it uses the controlled attenuators and phase shifters to feed each antenna of the array with the proper values of amplitude and phase. Bidirectional Amplifiers are embedded to the system to amplify the transmission and reception signals. They use constant values of amplification and, on this account, the attenuators have the role of fully adjusting the power amplitudes inserted in the antennas.

Controlled switches are used by the AAC to excite the correct Yagi-Uda antenna array among the three existing, depending on the remote device direction. Figure 8 illustrates the complete control system.

The system shall be able, from the geographical coordinates of the BTS and the device to be triggered, to automatically calculate the steering angle and thus polarize the antenna array to illuminate that direction with its maximum power. The geographical locations (latitude, longitude) of each system element shall be converted to a spherical coordinate system $(\Theta, \varphi)$. The BTS is located at a position equivalent to the point $(0,0)$ of this spherical coordinate system, and the reference of the angles is the vector that points in the normal direction of the antenna array.

Considering an element in the geographical position (lat, lon), the conversion to the system $(\Theta, \varphi)$ is given by

$$
\begin{aligned}
& \theta=\frac{\pi}{2}-\frac{\pi \text { lat }}{180}, \\
& \varphi= \begin{cases}\pi \cdot \frac{\text { lon }}{180}, & \text { if lon }>0, \\
2 \pi+\pi \cdot \frac{\text { lon }}{180}, & \text { otherwise. }\end{cases}
\end{aligned}
$$

Considering the BTS coordinates are $\left(\Theta_{\mathrm{BTS}}, \varphi_{\mathrm{BTS}}\right)$ and that the radius $R$ of the Earth remains approximately constant between small distances on its surface, the repositioning of the other devices $\left(\Theta_{\mathrm{dev}}, \varphi_{\mathrm{dev}}\right)$ in the system $(x, y$, $z$ ) so that the BTS is at the position equivalent to the geographical point (lat $=0$, long $=0$ ) is given by

$$
\left[\begin{array}{l}
x \\
y \\
z
\end{array}\right]=[A] \cdot\left[\begin{array}{c}
R \cos \varphi_{\mathrm{dev}} \sin \theta_{\mathrm{dev}} \\
R \sin \varphi_{\mathrm{dev}} \sin \theta_{\mathrm{dev}} \\
R \cos \theta_{\mathrm{dev}}
\end{array}\right]
$$

with $[\mathrm{A}]$ equal to

$$
\left[\begin{array}{ccc}
\sin \left(\theta_{\mathrm{BTS}}\right) \cos \left(\varphi_{\mathrm{BTS}}\right) & \sin \left(\theta_{\mathrm{BTS}}\right) \sin \left(\varphi_{\mathrm{BTS}}\right) & \cos \left(\theta_{\mathrm{BTS}}\right) \\
-\sin \left(\varphi_{\mathrm{BTS}}\right) & \cos \left(\varphi_{\mathrm{BTS}}\right) & 0 \\
-\cos \left(\theta_{\mathrm{BTS}}\right) \cos \left(\varphi_{\mathrm{BTS}}\right) & -\cos \left(\theta_{\mathrm{BTS}}\right) \sin \left(\varphi_{\mathrm{BTS}}\right) & \sin \left(\theta_{\mathrm{BTS}}\right)
\end{array}\right] .
$$

Finally, considering the vector $V_{r}$ as the zero-reference direction of the antenna array, the angle $\delta$ between $V_{r}$ and the device positioned at the point $P_{\mathrm{dev}}=(x, y, z)$ is given by

$$
\delta=\cos ^{-1}\left(\frac{V_{r} \cdot P_{\mathrm{dev}}}{V_{r} P_{\mathrm{dev}}}\right) .
$$

Figure 9 shows the complete flowchart of the process which obtains the proper excitation set to be applied into the array from the geographical coordinates of the network devices. The process starts with a command on the SCADA (Supervisory Control and Data Acquisition) system to access the device of interest. 


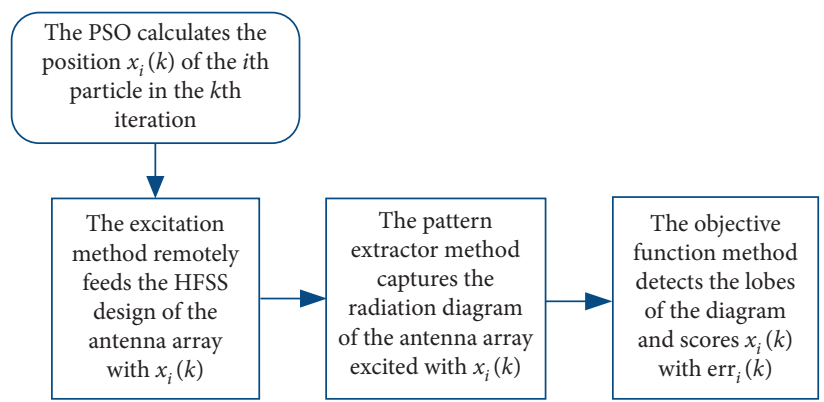

FIGURE 6: Flowchart of the algorithm process to remotely access the HFSS.

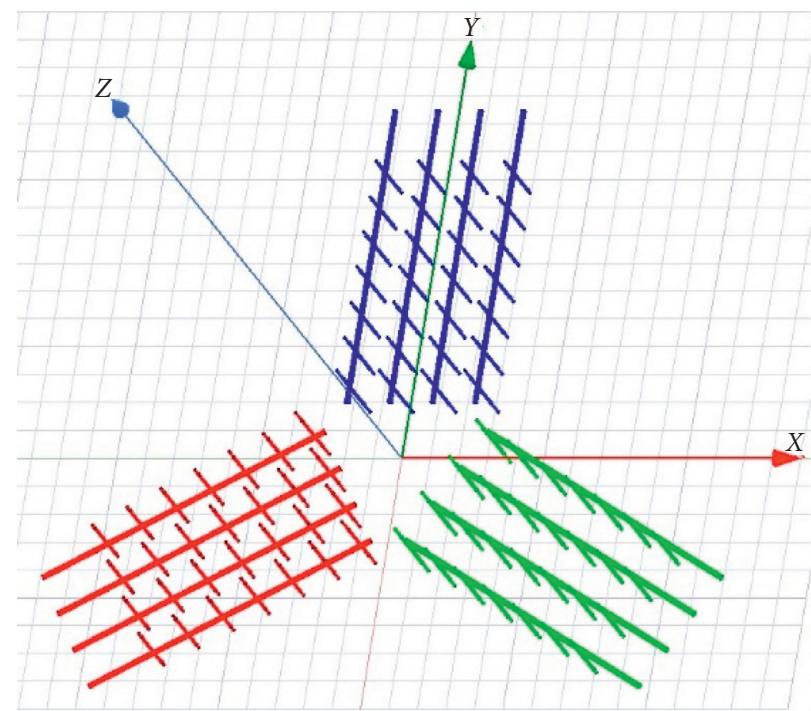

FIGURE 7: Intelligent array geometry configuration with $360^{\circ}$ coverage.

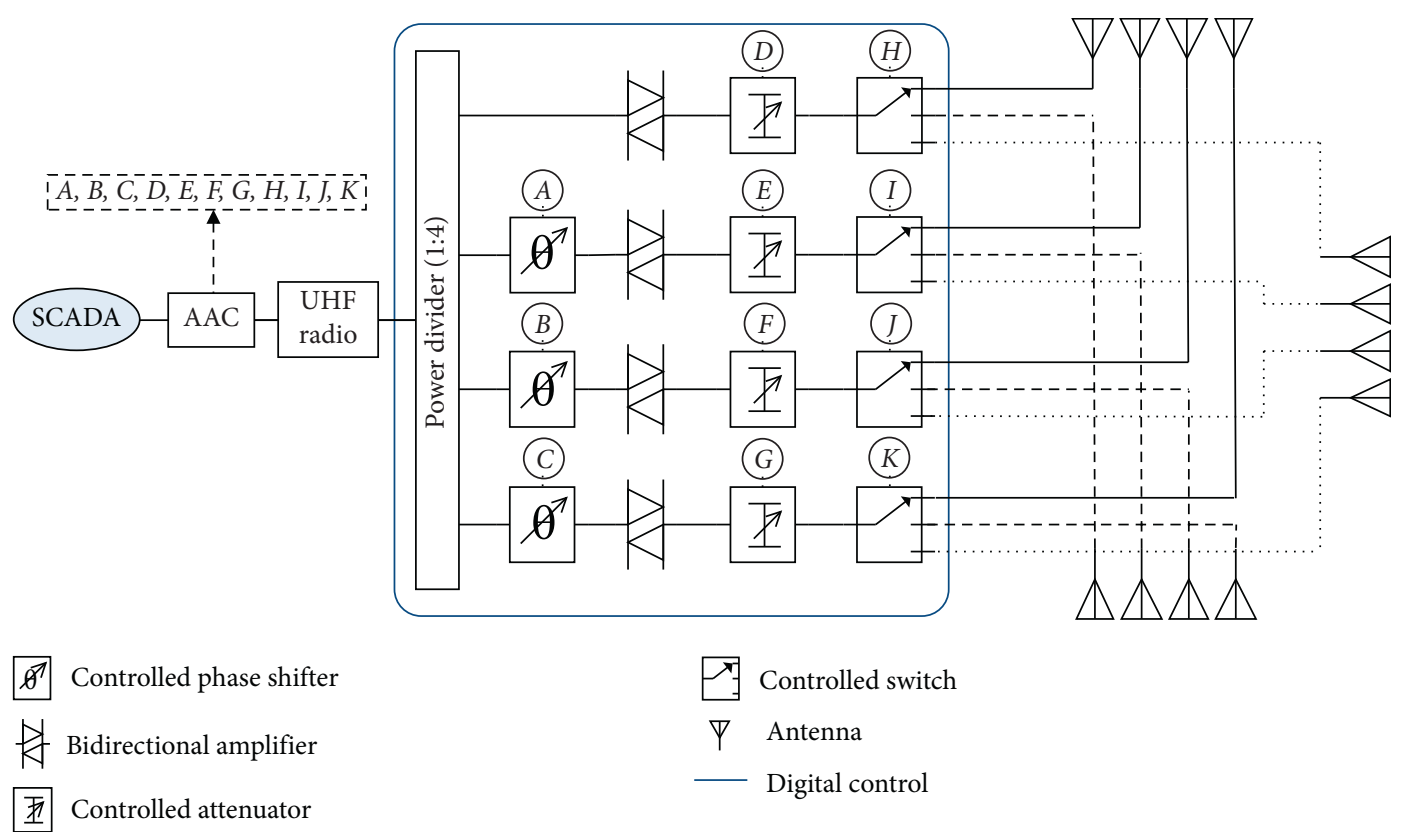

FiguRE 8: Complete system configuration for the antenna array. 


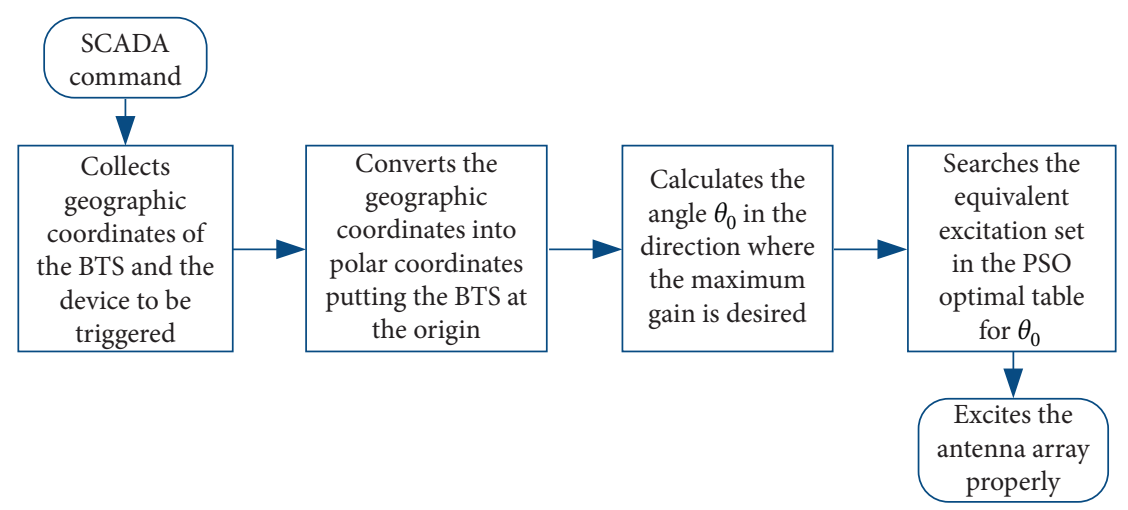

Figure 9: Flowchart of the process of phase shift calculation.

\section{Computational Results for PSO Optimization}

Attempts to adapt equation (4) to the Yagi-Uda antenna mathematical model and all its electromagnetic interferences end up generating a high computational cost. This cost includes the need to solve nonelementary integral equations of the impedances induced among the components of the model [25-28]. Furthermore, in some cases, there is no direct access to the modeling applied by the simulation software and its numerical approximations. That said, it would be relatively complicated to determine an efficient balance between computational cost and mathematical precision in those mathematical adaptations of equation (4). It should also be commented that the stochastic method uses the software model's own responses in its optimization, making it easily adapted to the system electromagnetic interferences particularities that are calculated by its platform. This indicates efficiency for different simulation software, within its own different levels of delivered numerical precision

The constructive geometry of the proposed smart array, based on the angular range of a $120^{\circ}$ azimuthal sector ensured by the PSO, consists of combining 3 intelligent collinear arrays, operating independently. The three arrays are mirrored, and each independently covers $120^{\circ}$, given a total coverage of $360^{\circ}$, as shown in Figure 7 .

Numerical simulations were made in HFSS [20] and Python [44] for the $0^{\circ}$ (Broadside), $30^{\circ}, 60^{\circ}$, and 90 (EndFire) configurations using PSO. Figure 10 shows the radiation diagram results of the three antenna array models. The positive direction of the $y$-axis in Figure 7 points to $90^{\circ}$ of Figure 10 patterns. For all the diagrams shown in this figure, the directivity is given in $\mathrm{dBi}$ for a frequency of 459.438 MHz.

\section{Complete System Simulation and Measured Results}

The calculation of the angle and distance of the device and relative to the BTS can be seen in Figure 11. The actual coordinates of a BTS $\left(8^{\circ} 11^{\prime} 51.4^{\prime \prime} \mathrm{S}, 35^{\circ} 34^{\prime} 39.7^{\prime \prime} \mathrm{W}\right)$ and a device $\left(8^{\circ} 12^{\prime} 51.7^{\prime \prime} \mathrm{S}, 35^{\circ} 33^{\prime} 30.6^{\prime \prime} \mathrm{W}\right)$ located in the city of Gravatá, state of Pernambuco, Brazil, were used. Comparing the calculated result with the measured one with Google Earth, it can be observed that the system has a relative error less than $0.2 \%$ in relation to the relative position of the elements.

The calculated angle in the conversion model for the BTS and one of the devices is $\theta_{0}=-42^{\circ}$. The result of the complete system is shown in Figure 12.

To validate the simulated mathematical model, the antenna array of Figure 13 was assembled with the 4 Yagi antennas of the numerical simulations, and the receiver antenna of Figure 14 was used to measure the power of the antenna array. The Antenna Array was rotated around its axis every $10^{\circ}$ to read the power at the receiver and thus build the radiation diagram of the array [19]. The measurements were performed considering the array parameters for the angles $\theta \_\mathrm{o}=10^{\circ}, \theta \_\mathrm{o}=30^{\circ}$ and $\theta \_\mathrm{o}=60^{\circ}$. A radio was connected to the array antenna and to the receiver antenna and the RSSI (Received Signal Strength Indication in $\mathrm{dBm}$ ) of the receiver antenna was captured via SSH (Secure Shell) protocol. Commercial attenuators and phase shifters were used to control the amplitude and phase of the signal.

Measurement parameters are as follows:

(i) Transmitted frequency of the array: $459 \mathrm{MHz}$

(ii) Distance between the fixed antenna and the array: $15.3 \mathrm{~m}$ (between the masts, bigger than the far-field distance)

(iii) Antenna height: $2 \mathrm{~m}$ (from the ground)

Figure 15 shows the comparison between the simulated and measured normalized radiation pattern. The results are in good agreement, and the differences observed between the measured and simulated responses are due to imperfect parallelism of the antennas and small errors in the phase and amplitude of the signals which feed the array of the experimental setup, compared to the calculated ones. However, the angle of maximum radiation matches accurately the desired positions of the elements, which demonstrates the capability of the system of automatically find the best set of parameters to excite the array and intelligently control the radiation pattern.

Table 1 shows the comparation of some smart antenna array technologies that use electrical excitation mechanisms for the control of the radiation pattern. The presented system 


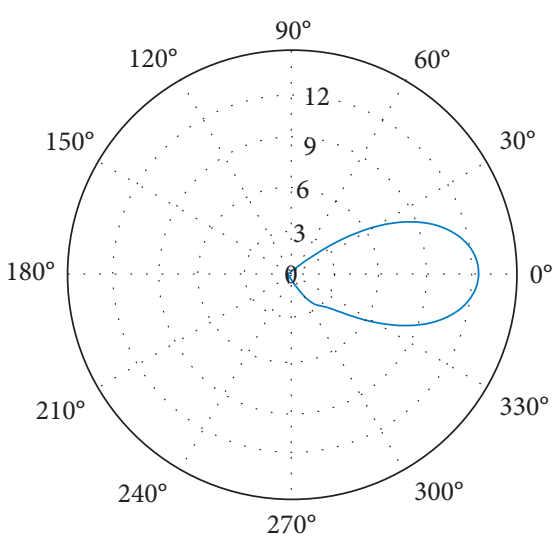

(a)

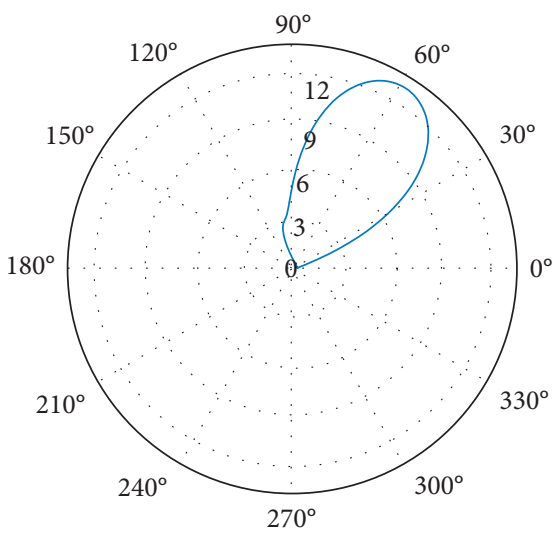

(c)

FIGURE 10: Radiation diagrams using PSO:

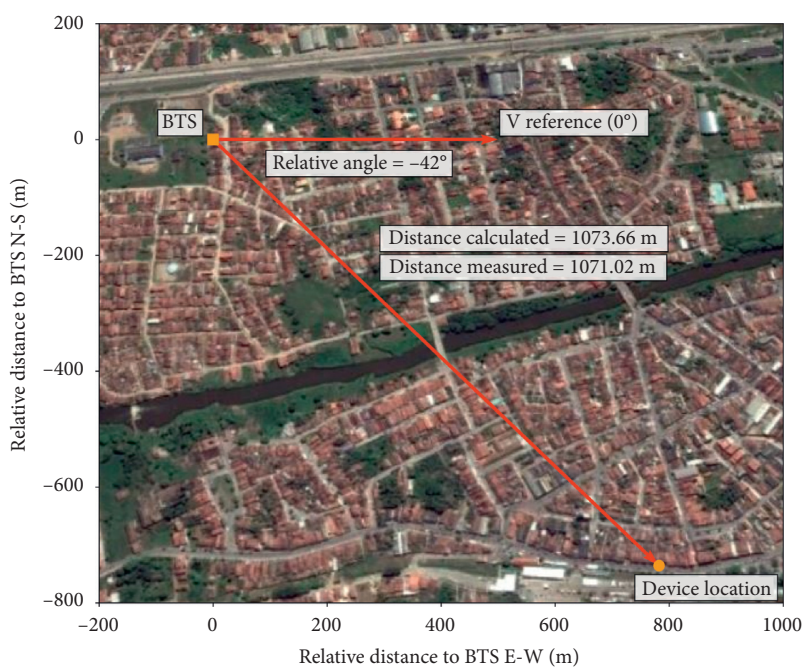

Figure 11: Satellite view of the test BTS and device location (by Google Earth) showing the angle and distance measured and evaluated by the system.

presents full angular coverage and no significant secondary beams. Additionally, the proposed method allows the manipulation of both phase and amplitude by an intelligent search PSO algorithm, in order to find independent and

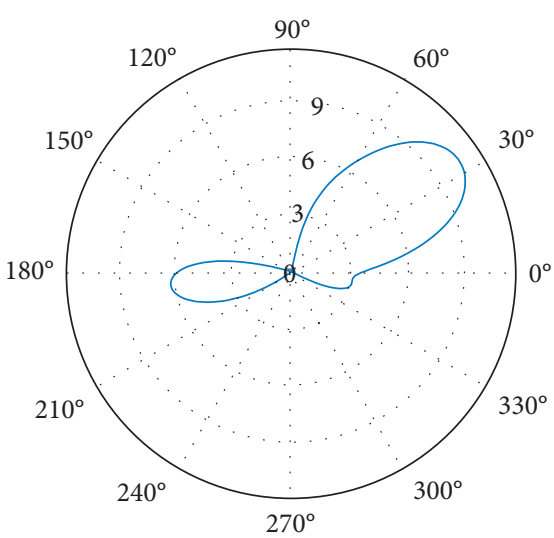

(b)

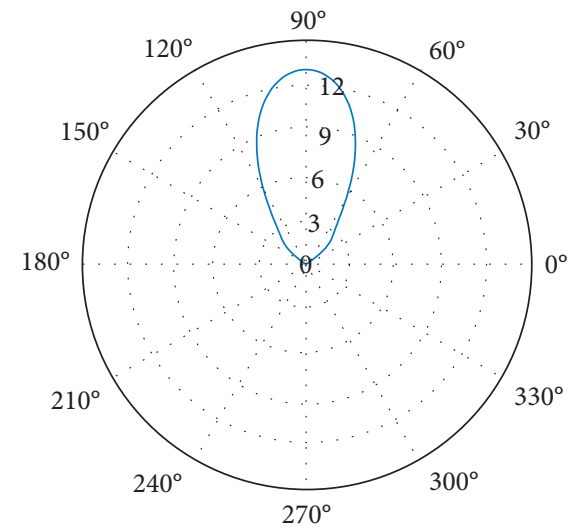

(d)

(b) $30^{\circ}-10.13 \mathrm{dBi}$, (c) $60^{\circ}-12.76 \mathrm{dBi}$, and (d) $90^{\circ}-13.31 \mathrm{dBi}$.

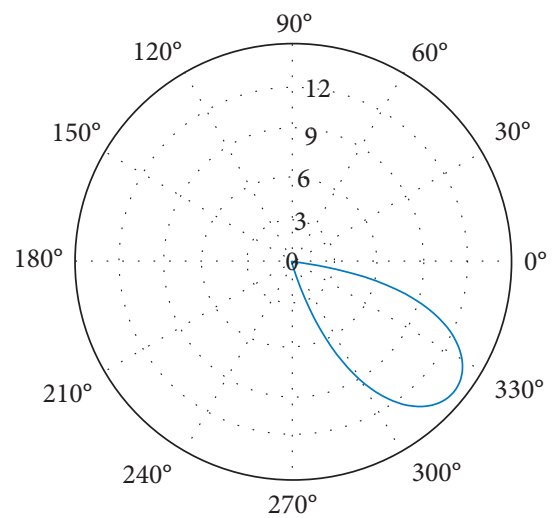

FIGURE 12: Radiation diagram for the BTS directing maximum power by method PSO $(14.00 \mathrm{dBi})$ to the test remote device $\left(-42^{\circ} \equiv 318^{\circ}\right)$. View in the plane azimuthal $(x-y)$.

optimized parameters to excite the arrays on each desired direction.

In order to numerically compare the advantages of the proposed scheme for the electrical distribution application, let us consider a typical BTS irradiating a signal in $459 \mathrm{MHz}$ with a power of $40 \mathrm{dBm}$, covering a circular area of radius $R$. Additionally, let us consider that remote devices connected to an $8 \mathrm{dBi}$ gain antenna are placed in the edge of this circle. 


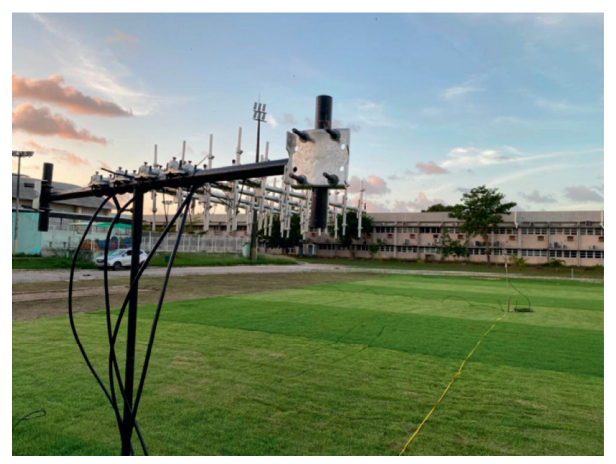

FIgURE 13: Antenna array.

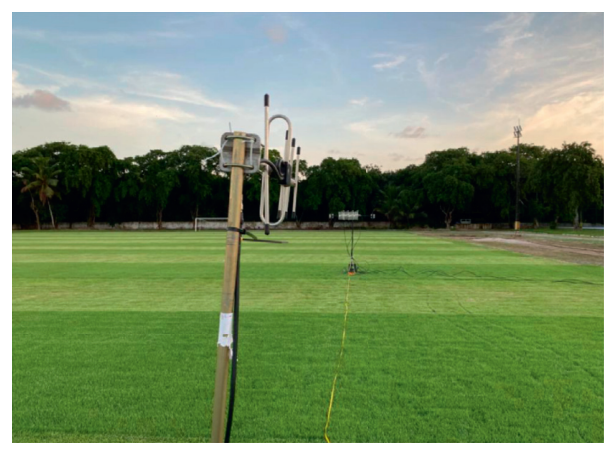

FIgURE 14: Receiver system.

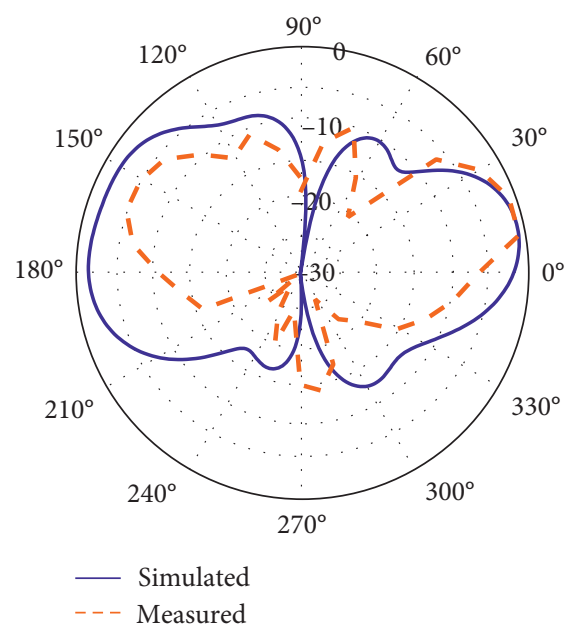

(a)

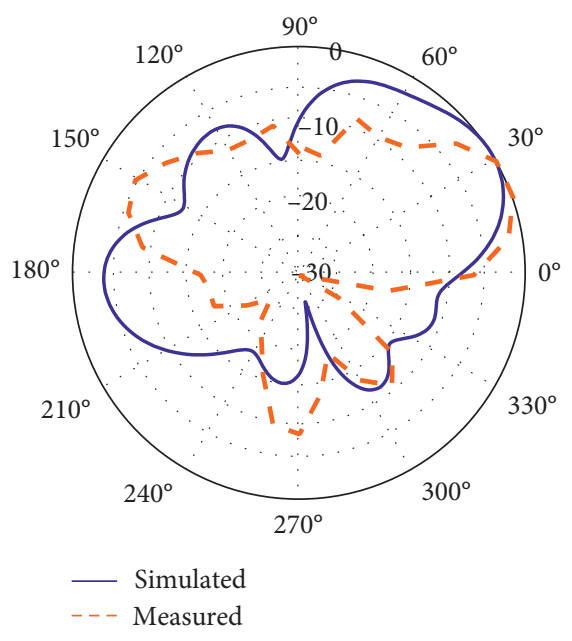

(b)

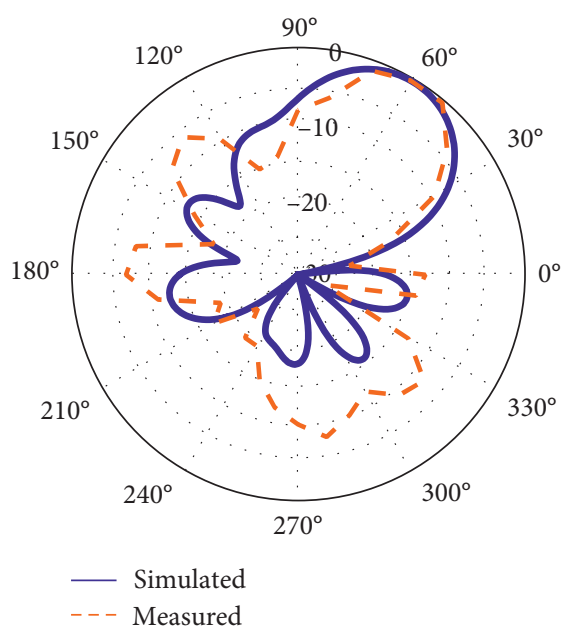

(c)

Figure 15: Measured results for (a) $\theta_{o}=10^{\circ}$, (b) $\theta_{o}=30^{\circ}$, and (c) $\theta_{o}=60^{\circ}$.

For the traditional collinear array $(6 \mathrm{dBi})$, applying the Frias equation [19] in an ideal situation where the BTS and remote devices are at the same height and there is no obstacle between them, the remote devices receive a power of $-53.77 \mathrm{dBm}$, at a distance equals to $R=9 \mathrm{~km}$ from the BTS. On the other hand, with the proposed scheme, the gain of the Yagi-Uda array ranges between 10.13 and $13.31 \mathrm{dBi}$. For this new scenario, in the worst case, where the gain is $10.13 \mathrm{dBi}$, a remote device receives the same power of $-53.77 \mathrm{dBm}$ at a further distance of $R=14.48 \mathrm{~km}$. Therefore, comparing the two situations, the antenna Yagi-Uda array covers a larger area $(659.7 \mathrm{~km} 2)$ than the colinear array $(254.46 \mathrm{~km} 2)$. In practical terms, 36 BTS with traditional colinear arrays would cover an area of $9,160.56 \mathrm{~km} 2$. Using the proposed scheme instead, to cover the same area, only 14 BTS with Yagi-Uda arrays are 
TABLE 1: Comparison between smart antenna arrays.

\begin{tabular}{|c|c|c|c|c|c|c|c|}
\hline \multirow{3}{*}{ Reference } & \multirow{3}{*}{ Technology } & \multicolumn{6}{|c|}{ Specifications } \\
\hline & & \multirow{2}{*}{$\begin{array}{c}\text { Freq } \\
(\mathrm{GHz})\end{array}$} & \multirow{2}{*}{$\begin{array}{l}\text { Angular } \\
\text { coverage }\end{array}$} & \multirow{2}{*}{$\begin{array}{c}\text { Significant secondary } \\
\text { beams }\end{array}$} & \multicolumn{3}{|c|}{ Controlled feed } \\
\hline & & & & & Phase & Amplitude & Method \\
\hline [19] & $\begin{array}{l}\text { Ten isotropic elements } \\
\text { linear }\end{array}$ & - & Full & Yes & Yes & Yes & $\begin{array}{c}\text { Binomial and } \\
\text { Chebyshev } \\
\text { polynomials }\end{array}$ \\
\hline [46] & $\begin{array}{l}\text { Six dipole switched } \\
\text { parasitic arrays }\end{array}$ & 3.5 & $60^{\circ}$ sector & No & Yes & No & $\begin{array}{c}\text { Genetic algorithm } \\
\text { (GA) }\end{array}$ \\
\hline [47] & Four linear dipole arrays & 2.16 & $180^{\circ}$ sector & Yes & Yes & No & $\begin{array}{l}\text { Dynamic adjustment } \\
\text { (Butler matrix) }\end{array}$ \\
\hline This work & $\begin{array}{l}\text { Three sets of four } \\
\text { Yagi-Uda linear arrays }\end{array}$ & 0.46 & Full & No & Yes & Yes & PSO \\
\hline
\end{tabular}

needed, a reduction of $61 \%$ in the number of BTS required for the same service.

\section{Conclusion}

This paper presented the design of an intelligent antenna array system using an optimized parameter search model in order to find the best amplitude and phase excitations to automatically direct the maximum radiation lobe towards any desired direction. It was shown that it is possible to automatically control the radiation pattern of the array according with the geographic coordinates of the transmitter and receiver, radiating the maximum power towards the devices connected to the communication link. The proposed optimized mathematical model is capable of feeding the set of antennas with the appropriate amplitude weights and excitation phases to generate a concentrated radiation lobe, allowing an efficient $360^{\circ}$ full coverage.

The optimization algorithm ensures, at a low computational cost, an optimal solution without it producing considerable secondary lobes in undesirable directions, which would force the system to share the density power of the output diagram with regions other than the one of interest. This allows the signal strength in the desired direction to be amplified to the maximum, resulting in a longer range of the radiated signal.

Compared to a configuration where the electrical parameters are static and the power is omnidirectionally radiated, the proposed smart antenna array system allows the coverage of a larger area, optimizing the maximum power delivery in either direction by simply varying intelligently the electrical excitations of the intelligent array.

\section{Data Availability}

The simulation data used to support the findings of this study are available from the corresponding author upon request. All the data shared in this paper have only academic interest and their reproduction is permitted unrestrictedly since the original paper is properly cited.

\section{Conflicts of Interest}

The authors declare that there are no conflicts of interest regarding the publication of this paper.

\section{Acknowledgments}

This work was supported by the Brazilian Electrical Power Agency Program (ANEEL) and Pernambuco State Electricity Company (CELPE), SA.

\section{References}

[1] R. Das, V. Madani, F. Aminifar et al., "Distribution automation strategies: evolution of technologies and the business case," IEEE Transactions on Smart Grid, vol. 6, no. 4, pp. 2166-2175, 2015.

[2] L. Tightiz, H. Yang, and M. J. Piran, "A survey on enhanced smart micro-grid management system with modern wireless technology contribution," Energies, vol. 13, no. 9, Article ID 2258, 2020.

[3] S. Alam, M. F. Sohail, S. A. Ghauri, I. M. Qureshi, and N. Aqdas, "Cognitive radio based smart grid communication network," Renewable and Sustainable Energy Reviews, vol. 72, pp. 535-548, 2017.

[4] N. Katic, "Performance Analysis of smart grid solutions in distribution power systems," Acta Polytechnica Hungarica, vol. 15, pp. 79-97, 2018.

[5] Q. Guo, J. Zhong, L. He, X. Guo, and Q. Zhang, "Analysis and design of wireless private network for electric power company," in Proceedings of the 2020 IEEE 5th Information Technology and Mechatronics Engineering Conference (ITOEC), pp. 736-740, Chongqing, China, June 2020.

[6] T. S. Rappaport and J. C. Liberti, Smart Antennas for Wireless Communications:IS-95 and Third Generation CDMA ApplicationsPrentice-Hall, Upper Saddle River, NJ, USA, 1999.

[7] M. Chryssomallis, "Smart antennas," IEEE Antennas and Propagation Magazine, vol. 42, no. 3, pp. 129-136, 2000.

[8] F. Luna, J. J. Durillo, A. J. Nebro, and E. Alba, "Evolutionary algorithms for solving the automatic cell planning problem: a survey," Engineering Optimization, vol. 42, no. 7, pp. 671-690, 2010.

[9] L. Azpilicueta, P López-Iturri, E Aguirre et al., "Analysis of radio wave propagation for ISM $2.4 \mathrm{GHz}$ wireless sensor networks in inhomogeneous vegetation environments," Sensors, vol. 14, no. 12, pp. 23650-23672, 2014.

[10] N. Faruk, S. I. Popoola, N. T. Surajudeen-Bakinde et al., "Path loss predictions in the VHF and UHF bands within urban environments: experimental investigation of empirical, heuristics and geospatial models," IEEE Access, vol. 7, pp. 77293-77307, 2019.

[11] T. Bai, R. Vaze, and R. W. Heath, "Analysis of blockage effects on urban cellular networks," IEEE Transactions on Wireless Communications, vol. 13, no. 9, pp. 5070-5083, 2014. 
[12] M. Hanaoui, H. Bouassam, M. Rifi, and H. Terchoune, "Improvement of energy efficiency of GSM BTS by using smart antenna system," Revue Méditerranéenne des Télécommunications, vol. 5, no. 2, 2015.

[13] L. C. Godara, "Applications of antenna arrays to mobile communications. I. Performance improvement, feasibility, and system considerations," Proceedings of the IEEE, vol. 85, no. 7, pp. 1031-1060, 1997.

[14] V. Ludwig-Barbosa, E. Schlosser, R. Machado, F. G. Ferreira, S. Müller Tolfo, and M. V. T. Heckler, "Linear array design with switched beams for wireless communications systems," International Journal of Antennas and Propagation, vol. 2015, Article ID 278160, 9 pages, 2015.

[15] Y. Harkouss, H. Shraim, and H. Bazzi, "Direction of arrival estimation for smart antenna in multipath environment using convolutional neural network," International Journal of $R F$ and Microwave Computer-Aided Engineering, vol. 28, Article ID e21282, 2018.

[16] S. Mohammadi, A. Ghani, and S. H. Sedighy, "Direction-ofArrival estimation in conformal microstrip patch array antenna," IEEE Transactions on Antennas and Propagation, vol. 66, no. 1, pp. 511-515, 2018.

[17] R. G. Ayestaron, "Fast near-field multifocusing of antenna arrays including element coupling using neural networks," IEEE Antennas Wirel. Propag. Lett, vol. 17, no. 7, pp. 12331237, 2018.

[18] D. K. Cheng, Field and Wave Electromagnetics, pp. 600-664, Pearson, London, UK, 2nd ed. edition, 2006.

[19] C. A. Balanis, Antenna Theory: Analysis and Design, pp. 283-321, John Wiley \& Sons, New York, NY, USA, 4th ed. edition, 2016.

[20] HFSS, 3D Electromagnetic Field Simulator for RF and Wireless Design, https://www.ansys.com/products/electronics/ansyshfss, 2020

[21] J. F. Lee, D. K. Sun, and Z. J. Cendes, "Tangential vector finite elements for electromagnetic field computation," IEEE Transactions on Magnetics, vol. 27, no. 5, pp. 4032-4035, 1991.

[22] R. F. Harrington, "The method of moments in electromagnetics," Journal of Electromagnetic Waves and Applications, vol. 1, no. 3, pp. 181-200, 1987.

[23] M. J. L. Alves and M. Sampaio de Alencar, "Effects of mutual coupling in smart antenna arrays," in 2007 SBMO/IEEE MTT$S$ International Microwave and Optoelectronics Conference, pp. 418-421, Salvador, Brazil, November 2007.

[24] B. Liao and S.C. Chan, "Adaptive beamforming for uniform linear arrays with unknown mutual coupling," IEEE Antennas and Wireless Propagation Letters, vol. 11, pp. 464-467, 2012.

[25] T. A. Milligan, Modern Antenna Design, pp. 485-497, John Wiley \& Sons, Hoboken, NJ, USA, 2nd ed. edition, 2005.

[26] J.-S. Jian-She Wang, "Finite element computation of antenna impedance including feed modeling," IEEE Transactions on Magnetics, vol. 33, no. 2, pp. 1500-1503, 1997.

[27] W. Ma, M. Rayner, and C. Parini, "The modelling of a Yagi-Uda array antenna using the DGF-FDTD method," in Proceedings of the IEEE Antennas and Propagation Society International Symposium. Digest. Held in conjunction with: USNC/CNC/URSI North American Radio Sci. Meeting (Cat. No.03CH37450), vol. 3, pp. 780-783, Columbus, OH, USA, June 2003.

[28] S. Pandav and G. Kumar, "Modeling of yagi-uda antenna using method of moments," IETE Technical Review, vol. 17, no. 5, pp. 283-291, 2015.

[29] M. Khodier and M. Al-Aqil, "Design and optimisation of Yagi-Uda antenna arrays," IET Microwaves, Antennas and Propagation, vol. 4, no. 4, pp. 426-436, 2010.
[30] M. Rattan and B. S. Manjeet Singh Patterh, "Sohi, "optimization of gain, impedance, and bandwidth of yagi-uda array using particle swarm optimization"' International Journal of Antennas and Propagation, vol. 2008, p. 4, Article ID 193242, 2008.

[31] E. A. Jones and W. T. Joines, "Design of Yagi-Uda antennas using genetic algorithms," IEEE Transactions on Antennas and Propagation, vol. 45, no. 9, pp. 1386-1392, 1997.

[32] L. A. Greda, A. Winterstein, D. L. Lemes, and M. V. T. Heckler, "Beamsteering and beamshaping using a linear antenna array based on particle swarm optimization," IEEE Access, vol. 7, pp. 141562-141573, 2019.

[33] X. Zhang, D. Lu, X. Zhang, and Y. Wang, "Antenna array design by a contraction adaptive particle swarm optimization algorithm," EURASIP Journal on Wireless Communications and Networking, vol. 2019, no. 1, p. 57, 2019.

[34] J. Kennedy and R. Eberhart, "Particle swarm optimization," in Proceedings of ICNN'95-International Conference on Neural Networks, vol. 4, pp. 1942-1948, Perth, WA, Australia, December 1995.

[35] A. Ratnaweera, S. K. Halgamuge, and H. C. Watson, "Selforganizing hierarchical particle swarm optimizer with timevarying acceleration coefficients," IEEE Transactions on Evolutionary Computation, vol. 8, no. 3, pp. 240-255, 2004.

[36] S. Xu and Y. Rahmat-Samii, "Boundary conditions in particle swarm optimization revisited," IEEE Transactions on Antennas and Propagation, vol. 55, no. 3, pp. 760-765, 2007.

[37] C. Han and L. Wang, "Array pattern synthesis using particle swarm optimization with dynamic inertia weight," International Journal of Antennas and Propagation, vol. 2016, Article ID 1829458, 7 pages, 2016.

[38] D. Liu, Q. Feng, W.-B. Wang, and X. Yu, "Synthesis of unequally spaced antenna arrays by using inheritance learning particle swarm optimization," Progress In Electromagnetics Research, vol. 118, pp. 205-221, 2011.

[39] S. Huang, L. Yu, F. Han, and Y. Luo, "Pipeline implementation of polyphase PSO for adaptive beamforming algorithm," Wireless Communications and Mobile Computing, vol. 2017, Article ID 3926821, 12 pages, 2017.

[40] S. K. Goudos, C. Kalialakis, and R. Mittra, "Evolutionary algorithms applied to antennas and propagation: a review of state of the art," International Journal of Antennas and Propagation, vol. 2016, Article ID 1010459, 12 pages, 2016.

[41] J. Robinson and Y. Rahmat-Samii, "Particle swarm optimization in electromagnetics," IEEE Transactions on Antennas and Propagation, vol. 52, no. 2, pp. 397-407, 2004.

[42] N. Jin and Y. Rahmat-Samii, "Particle swarm optimization for antenna designs in engineering electromagnetics," Journal of Artificial Evolution and Applications, vol. 2008, Article ID 728929, 10 pages, 2008.

[43] G. Sun, L. Yanheng, L. Han, L. Shuang, W. Aimin, and L. Boyu, "An antenna array sidelobe level reduction approach through invasive weed optimization," International Journal of Antennas and Propagation, vol. 2018, 2018.

[44] Python, https://www.python.org/, 2015.

[45] M. R. Santos, B. G. M. de Oliveira, M. T. de Melo et al., "A smart antenna system for remote supervision," International Journal of Applied Electromagnetics and Mechanics, vol. 45, no. 1-4, pp. 293-299, 2014.

[46] A. I. Sotiriou, P. K. Varlamos, P. T. Trakadas, and C. N. Capsalis, "Broadband wireless access base station performance using smart antenna cell," in Proceedings of the 2007 International workshop on Antenna Technology: Small and Smart Antennas Metamaterials and Applications, pp. 357-360, Cambridge, UK, March 2007. 
[47] F. Bobor-Oyibo, S. J. Foti, and D. Smith, "A multiple switched beam Smart antenna with beam shaping for dynamic optimisation of capacity \& coverage in mobile telecommunication networks," in Proceedings of the 2008 8th International Symposium on Antennas, Propagation and EM Theory, pp. 356-359, Kunming, China, November 2008. 